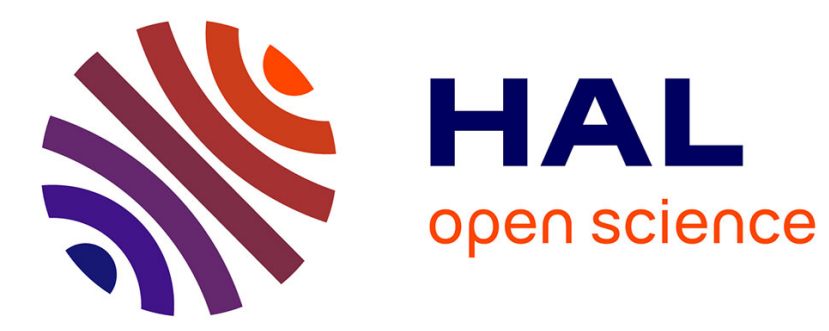

\title{
La Pensée Comme Expérience Vécue
}

Janette Friedrich

\section{To cite this version:}

Janette Friedrich. La Pensée Comme Expérience Vécue. Revue de Synthèse, 2010, 131 (1), pp.53-75. 10.1007/s11873-009-0108-1 . hal-00578392

\section{HAL Id: hal-00578392 \\ https://hal.science/hal-00578392}

Submitted on 20 Mar 2011

HAL is a multi-disciplinary open access archive for the deposit and dissemination of scientific research documents, whether they are published or not. The documents may come from teaching and research institutions in France or abroad, or from public or private research centers.
L'archive ouverte pluridisciplinaire HAL, est destinée au dépôt et à la diffusion de documents scientifiques de niveau recherche, publiés ou non, émanant des établissements d'enseignement et de recherche français ou étrangers, des laboratoires publics ou privés. 


\title{
LA PENSÉE COMME EXPÉRIENCE VÉCUE L'école de Würzbourg
}

\author{
Janette FRIEDRICH*
}

\begin{abstract}
RÉSUMÉ: Le centre de l'article est consacré à la psychologie de la pensée élaborée par l'école de Würzbourg au début du $\mathrm{Xx}^{\mathrm{e}}$ siècle. Il s'agit ici de montrer que cette école articule deux mouvements contemporains: le projet d'une psychologie d'un point de vue empirique de Brentano, et l'emploi de l'expérimentation au-delà de la sphère des perceptions. À partir des travaux de Karl Bühler est mise en question la légitimité de l'approche du psychologisme appliquée à toutes les écoles psychologiques participant à cette époque au débat sur la nature de la pensée. L'idée bühlerienne d'un savoir actuel ayant pour but d'éviter un mentalisme localisationniste, si attirant pour une psychologie de la pensée, est également discutée.
\end{abstract}

Mots-CLÉs: psychologie de la pensée, introspection, psychologisme, savoir (actuel).

\section{THOUGHT AS LIVED EXPERIENCE The Würzburg School}

ABSTRACT: The core of our essay is the "psychology of thought" (Denkpsychologie), which was elaborated by the Würzburg School at the beginning of the $20^{\text {th }}$ century. We will show how this School synthesized two contemporary trends: Brentano's project of a psychology from an empirical point of view and the use of experiments beyond the limits of perception. Through the works of Karl Bühler, we will criticize the legitimacy of the psychologistic approach, which was applied to all psychological schools of thought participating in the debate on the nature of thought. We will also discuss the Bühlerian idea of a present knowledge, which aims to avoid the consequence of mind localization, a very tempting issue for the psychology of thought.

KEYWORDS: psychology of thought, introspection, psychologistic approach, (present) knowledge.

\footnotetext{
* Janette Friedrich, née en 1961, est philosophe. Directrice de programme au Collège international de philosophie et membre du laboratoire " Histoire et épistémologie des sciences du langage » du CNRS, elle travaille à la faculté de psychologie et des sciences de l'éducation de l'université de Genève. Ses recherches portent sur l'histoire et l'épistémologie des sciences de l'homme, notamment sur la tradition soviétique des années 1920 et les penseurs allemands (Kurt Goldstein, Alfred Schütz, Karl Bühler). Elle a notamment édité La Signification historique de la crise en psychologie de Lev Vygotskij (Paris, Delachaux et Niestlé, 1999) et La Théorie du langage de Karl Bühler (Marseille, Agone, 2009).

Adresse: Université de Genève, Faculté de psychologie et des sciences de l'éducation, 40, boulevard du Pont d'Arve, CH-1211 Genève 4.

Courrier électronique: Janette.Friedrich@unige.ch
} 


\section{DAS DENKEN ALS ERLEBNIS \\ Die Würzburger Schule}

ZuSAMmenfaSSUNG: Der Artikel behandelt die zu Anfang des 20. Jahrhunderts von der Würzburger Schule ausgearbeitete Denkpsychologie. Es wird gezeigt wie diese Schule zwei zeitgenössische Strömungen zusammenführt: zum einen Brentanos Projekt einer Psychologie vom empirischen Standpunkt, zum anderen die Anwendung experimenteller Methoden über den Bereich der Wahrnehmungen hinaus. Ausgehend von den Arbeiten Karl Bühlers wird die Legitimität des Psychologismusvorwurfs hinterfragt, der zu dieser Zeit auf alle an der Debatte um das Wesen des Denkens beteiligten psychologischen Schulen angewendet wurde. Ebenso wird Bühlers Konzeption des aktuellen Wissens vorgestellt, mit der er den lokalistischen Mentalismus zu vermeiden sucht, der nach wie vor für die Denkpsychologie attraktiv erscheint.

STICHWÖRTER: Denkpsychologie, Introspektion, Psychologimus, aktuelles Wissen.

$$
\text { جانات فريديريخ : الفكر كتجربة معيشة، مدرسة فورزبورغ. }
$$

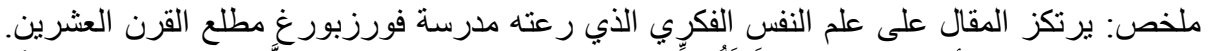

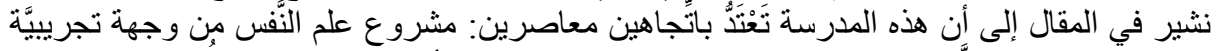

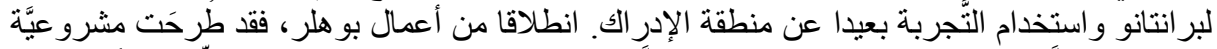

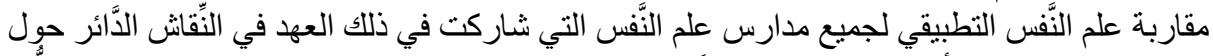

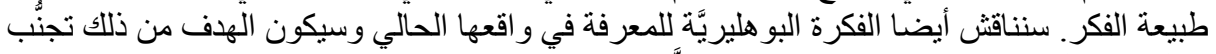
عقليَّة مركزيَّة بصفتها من تجذب الفئب علم الفكر النَّفسي. الكلمات المفاتيح: علم الفكر النَّفس، استبطان، علم النفس النفسي، معرفة حاليَّة.

実際経験としての思考一ヴュルツブルク学派ジャネット・フレデリッヒ

要約 : 本文は 20 世紀のヴュルツブルク学派によって研究された思考の心理学

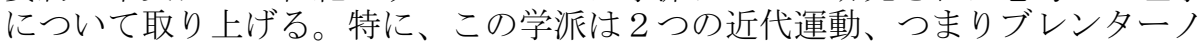
による経験的立場からの心理学に挙げられる研究、そして知覚の範囲を越えた 実験の採用、と関連しているということを取り上げたい。ビューラーの業績か ら、当時、思考の性質について議論していたすべての心理学派の心理学主義ア プローチの正当性が問われるようになった。本文ではさらに、思考心理学にと って魅力的だった位置的精神主義を避けるために、ビューラー派が考え出した 現在知の理念についても検討したい。

キーワード：思考心理学、内省、心理主義、（現在）知 
$\mathrm{L}$ e $\mathrm{Xx}^{\mathrm{e}}$ siècle s'est caractérisé par une ascension ininterrompue des recherches sur les processus cognitifs et mentaux dont on supposait quasi automatiquement que le siège serait à chercher dans le cerveau ou, selon la formule plus classique, "dans l'esprit ». Mais ce même siècle a vu également apparaître une critique importante de ce mythe de l'intériorité si difficile à combattre dans des disciplines comme la psychologie, et notamment dans la psychologie de la pensée qui nous intéressera davantage ici. Ainsi Christiane Chauviré rapporte, dans un de ses textes sur Peirce, que déjà « pour le jeune Peirce, une pensée ne saurait être instantanément présente à l'esprit; une pensée n'est rien que nous puissions avoir dans l'esprit $^{1} »$, ce qui conduit au problème suivant: où faudrait-il donc chercher ce fameux « lieu de la pensée »? D'autres auteurs ont évoqué ce même problème. Gilbert Ryle nous met par exemple en garde, dans La Notion d'esprit:

«Il est possible et même souhaitable d'éviter l'expression "en esprit" car elle habitue ceux qui l'emploient à penser que les esprits sont des "endroits" bizarres qu'habitent des phantasmes jouissant d'un statut particulier. Le but de mon livre est, en partie, de montrer que l'exercice des qualités de l'esprit n'a qu'exceptionnellement lieu "dans la tête", au sens ordinaire de cette expression, et que ces exceptions n'ont aucune priorité sur les autres ${ }^{2}$.»

Ludwig Wittgenstein, quant à lui, applique à la compréhension la même analyse que Peirce à la pensée: "Il doit y avoir davantage dans "Il comprend" que: La formule lui vient à l'esprit ${ }^{3}$. » Alors s'il est vrai, comme l'affirment Peirce, Ryle, Wittgenstein et bien d'autres, que la pensée n'est pas à identifier à un contenu mental ou à une activité consciente, qu'est-ce en fin de compte que ce plus, ce davantage qui rend justement impossible de localiser la pensée à l'intérieur, qu'il s'agisse de l'esprit ou du cerveau? Les réflexions que Wittgenstein avance dans les Recherches philosophiques à propos de la compréhension nous semblent valables aussi pour la pensée, les deux phénomènes étant interchangeables, et ont le mérite d'apporter une première réponse à cette question. Wittgenstein refuse catégoriquement de voir dans la compréhension un processus psychique:

« Ne conçois surtout pas la compréhension comme un "processus psychique"! - Une telle façon de parler te plonge en effet dans la confusion. [...] Au sens où il y a des processus caractéristiques de la compréhension (y compris des processus psychiques), la compréhension n'est pas elle-même un processus psychique. (La diminution ou l'intensification d'une sensation de douleur, l'écoute d'une mélodie, d'une phrase: ce sont des processus psychiques) $)^{4}$. »

Si l'on prend au sérieux les exemples que Wittgenstein avance quand il parle des phénomènes psychiques, pour en distinguer fermement la compréhension, on a l'impression de retrouver le débat caractéristique de la période de la naissance de la

\footnotetext{
1. Chauviré, 2003, p. 279.

2. RYLE, 1949, ici 2005, p. 112.

3. Wittgenstein, 1953, ici 2004, p. 100.

4. Wittgenstein, 1953, ici 2004, p. 101.
} 
psychologie scientifique. Son refus de traiter la compréhension comme un phénomène psychique pourrait être interprété comme une autre manière de dire ce que la psychologie moderne au début $\mathrm{du} \mathrm{xx}^{\mathrm{e}}$ siècle ne cesse de répéter: le jugement, le raisonnement, la pensée, la mémoire sont des phénomènes difficiles à transformer en objets d'une psychologie expérimentale, ou pour le dire d'une manière plus moderne, sont des phénomènes à l'égard desquels la naturalisation échoue nécessairement. Tandis que la sensation d'une douleur, la perception d'un son sont mesurables avec des appareils spécialement conçus dans ce but, les phénomènes psychiques supérieurs, comme les psychologues les désignaient, ne se prêtent pas à une telle analyse objectivante. Or, Wilhelm Wundt, le père de la psychologie scientifique, en a conclu que la pensée, le jugement, etc., devraient faire l'objet d'un autre type de psychologie, à laquelle il attribua le terme un peu trompeur de Völkerpsychologie (psychologie du peuple). Au sein de cette psychologie, les phénomènes psychiques sont analysés sous la forme de produits objectivés: ce sont des mythes, des religions ou des langues qui se trouvent au centre de l'intérêt, ce qui rapproche cette psychologie du projet des sciences de l'esprit tel qu'il a été développé en philosophie par Wilhelm Dilthey ${ }^{5}$.

L'aspect qui nous intéresse ici n'est cependant pas cette tentative d' «extraire » la pensée, le jugement, la compréhension du domaine proprement psychologique. Il s'agit en revanche d'analyser une des premières tentatives d'application à la pensée d'une démarche dite empirique, de transformation de la pensée en objet d'une psychologie expérimentale afin de montrer qu'il est possible de saisir la pensée comme un phénomène psychique en évitant l'alternative peu féconde entre une description physico-physiologique et une description mentaliste de celle-ci. Il s'agit de l'école de Würzbourg, désignation courante en histoire de la psychologie pour résumer des recherches concernant la pensée, le jugement et la volonté entreprises entre 1894 et 1909 sous la direction d'Oswald Külpe, professeur ordinaire de philosophie et d'esthétique et fondateur de l'Institut de psychologie à l'université de Würzbourg ${ }^{6}$. Dès le début, les réactions à cette nouvelle Psychologie de la pensée sont partagées. Dans les manuels actuels, on souligne encore que ces recherches ont bouleversé la théorie psychologique de la pensée au début du $\mathrm{XX}^{\mathrm{e}}$ siècle, tout en mettant en doute le fait qu'elles aient pu vraiment correspondre aux critères de scientificité importés dans la jeune psychologie en voie de constitution à partir des sciences de la nature. Tandis qu'entre 1925 et 1960 leurs recherches se trouvent peu convoquées, les références positives s'accumulent à partir des années 1970 et certains auteurs vont jusqu'à dire que les chercheurs de l'école de Würzbourg sont des précurseurs des sciences cognitives. Mais ce n'est pas l'histoire de la réception de cette école qui sera présentée ici ${ }^{7}$. L'objectif est de démontrer que ses membres partagent un certain nombre d'idées anti-mentalistes à propos de la pensée qui ressemblent à celles développées plus tard au sein de la philosophie par Wittgenstein et par Ryle. La thèse principale est ici la suivante: en proposant une analyse de la pensée qui se veut exclusivement psychologique, les chercheurs de Würzbourg découvrent certaines propriétés de la pensée permettant d'argumenter en faveur d'une théorie anti-mentaliste de l'esprit.

5. Cette conception est notamment résumée dans DiLThey, 1910, ici 1988.

6. Voir, sur l'histoire de l'école de Würzbourg, JAHNKE et SCHNEIDER, 1999.

7. Voir, sur l'histoire de la réception, AsH, 1999; EschBACH, 1997. 
Pour prouver cette thèse, on se limitera aux travaux de Karl Bühler (1879-1963), assistant de Külpe à Würzbourg. Külpe, qui a été formé par Wundt et gardera durant toute sa vie une relation loyale vis-à-vis de son maître en atténuant des divergences théoriques rapidement apparues, n'a écrit que deux textes qui portent explicitement sur la psychologie de la pensée. Les travaux les plus connus de son école ont été réalisés par Karl Marbe (1869-1953), Narziss Ach (1871-1946), Otto Selz (1881-1943), August Messer (1867-1937) et Henry Watt (1879-1925). C'est la publication des deux premières parties de l'habilitation de Bühler intitulée «Tatsachen und Probleme zu einer Psychologie der Denkvorgänge » (Faits et problèmes pour une psychologie des processus de la pensée) qui déclenche le débat avec le père de la psychologie allemande. Wundt réagit en 1907 avec un article de presque soixante pages, intitulé « Über Ausfrageexperimente und über die Methoden zur Psychologie des Denkens » (Sur les expériences par questionnement et sur les méthodes pour une psychologie de la pensée), auquel Bühler répond promptement dans un texte qu'il ajoute à la publication de son habilitation ${ }^{8}$. Wundt riposte avec un deuxième texte beaucoup plus court et clôt le débat, sans prendre en compte les arguments avancés par Bühler ${ }^{9}$. Dans ce qui suit, l'on proposera une analyse des recherches développées par Bühler concernant la conception du psychique, analyse ensuite étayée par quelques références précises aux travaux ultérieurs de ce même auteur.

\section{UNE PSYCHOLOGIE EMPIRIQUE - EXPÉRIMENTALE ET EXPÉRIENTIELLE}

Parler d'une psychologie empirique pour les travaux de Würzbourg demande deux précisions. Premièrement, les chercheurs de Würzbourg se voient dans la continuation d'une psychologie expérimentale telle qu'elle a été conçue par Wundt avec un point de désaccord, à savoir que les fonctions psychiques dites supérieures sont considérées par eux comme des phénomènes dont on pourrait produire une connaissance à l'aide de dispositifs expérimentaux. Il faut rappeler que pour Wundt le domaine de ces fonctions était un terrain interdit à l'expérimentation, réservée quant à elle aux phénomènes des sensations et des perceptions facilement objectivables. Deuxièmement, une des sources de cette psychologie de la pensée est sans aucun doute à chercher dans le projet d'une psychologie descriptive élaboré par Franz Brentano et propagé d'une manière plus ou moins fidèle par ses étudiants, Stumpf, Husserl, Meinong et autres. Ainsi Bühler a suivi une année les enseignements de Stumpf à l'université de Berlin et sa lecture des Recherches logiques de Husserl se fait sentir tout au long de son habilitation. L'envoi de cette dernière à Meinong a d'ailleurs suscité une correspondance entre les deux penseurs qui a duré jusqu'en $1918^{10}$. C'est la perception interne, véritable pivot de la doctrine de Brentano et considérée comme le seul moyen pour étudier les phénomènes psychiques, qui acquiert pour les würzbourgiens le caractère d'une prémisse fondamentale, laquelle n'est pas à questionner, même si les références directes

8. Voir BÜHLER, 1908b.

9. Voir WundT, 1908.

10. La correspondance entre Karl Bühler et Alexius Meinong se trouve dans VonK, 1992, p. 273-292. 
à Brentano se font rares dans leurs écrits. En concevant un dispositif expérimental basé exclusivement sur l'introspection, l'école de Würzbourg est une des premières à réaliser ce que Brentano postule comme démarche privilégiée en psychologie:

«Comme les sciences de la nature, la psychologie repose sur la perception et sur l'expérience. Mais sa source essentielle est la perception interne de nos propres phénomènes psychiques. En quoi consistent une représentation, un jugement [...] nous ne le saurions jamais, si la perception interne de nos propres phénomènes ne nous l'apprenait ${ }^{11}$. »

«La perception interne de nos propres phénomènes psychiques est donc la première source des expériences indispensables aux recherches psychologiques ${ }^{12} . »$

La méthode utilisée à Würzbourg a été désignée sous le terme d'introspection contrôlée et le dispositif dans lequel elle est employée est décrit par Bühler comme suit:

«La méthode employée dans ces recherches consiste à faire penser quelqu'un à quelque chose et, immédiatement après, à lui faire décrire exactement la façon dont s'est déroulée sa pensée; puis on fait varier le "quelque chose", suivant les principes expérimentaux; voilà la méthode sous sa forme la plus simple ${ }^{13}$. »

Il s'agit donc d'un recours à la phénoménalité des processus mentaux, au fonctionnement de l'esprit tel qu'il a été vécu et rapporté par le sujet. Les questions adressées aux sujets des expérimentations dans les recherches d'Ach, de Marbe et de Bühler sont toutes formulées de la même façon et visent justement à dégager le vécu de la pensée et du jugement: "Quels ont été les phénomènes que vous avez éprouvés dans la conscience pendant la résolution de la tâche? Qu'est-ce que nous vivons lorsque nous pensons ${ }^{14}$ ? \ Dans ces recherches, la pensée a été intentionnellement provoquée et contrôlée à travers des exercices construits par les chercheurs et analysée dans des conditions artificiellement modifiées, ce qui légitime, selon Bühler, de parler d'expérimentations dans le sens qu'on donne à ce terme à cette époque. Rappelons que les expérimentations telles qu'elles ont été réalisées et déclarées comme exemplaires par Wundt comprendraient normalement trois étapes: 1) la présentation du stimulus

11. Brentano, 1874, ici 2008, p. 42.

12. Brentano, 1874, ici 2008, p. 47.

13. BÜHLER, 1907a, p. 376-377.

14. Une présentation détaillée des recherches de Watt, Messer et Bühler se trouve dans BurLoud, 1927. Dans le dispositif de Bühler, l'expérimentateur lit un énoncé qui présente soit un aphorisme philosophique ou poétique, soit un problème philosophique complexe et pose la question: «Comprenez-vous? » ou « Est-il exact que...? » ou encore « Saisissez-vous comment on peut être amené à dire que... ? » (BüHLER, 1907b, p. 311, cité par Burloud, 1927, p. 118). Parmi les problèmes philosophiques, on trouve des énoncés comme suit: «Le Moyen Âge a-t-il connu le théorème de Pythagore? »; «Est-ce que Eucken a raison, lorsqu'il dit que l'homme ne peut prendre conscience des limites de la connaissance que s'il les dépasse? » (BüHLER, 1907b, p. 304). Les aphorismes du genre «Donnez à chacun ce qui lui revient serait souhaiter la justice et créer le chaos » (BüHLER, 1907b, p. 311, cité d'après BurLOUD, 1927, p. 117 f) ont été trouvés chez Nietzsche, Rückert et d'autres auteurs contemporains. 
(par un appareil), 2) le temps qui s'écoule avant la réaction (mesuré par des appareils comme le chronomètre), 3) la réaction du sujet sous la forme d'une trace enregistrée par un appareil. Cette triple structure se retrouve bel et bien dans les expériences qui se sont déroulées à Würzbourg, avec deux modifications apparemment minimes : la place de l'appareil qui sert de stimulus est occupée par le chercheur qui propose les tâches au sujet, tandis que les réactions ne sont pas « enregistrées » par un appareil mais sous forme de verbalisations rapportées ${ }^{15}$.

Pourtant remettre au goût du jour l'introspection, même à l'intérieur d'un cadre dit expérimental, a été et est encore aujourd'hui une entreprise risquée. En se référant aux critères de scientificité, Wundt attaque dans ses deux articles adressés à Bühler avant tout cette méthode, et en dépit d'un intérêt renouvelé pour la psychologie de la pensée de Würzbourg, force est de constater qu' " on a laissé de côté l'introspection provoquée et la conviction sur laquelle elle se basait, à savoir qu'une observation directe des processus psychiques serait possible ${ }^{16} \gg$. La disqualification de ce mode d'accès aux faits psychologiques se présente comme un lieu commun et hante encore et encore les interprétations des recherches de Würzbourg ${ }^{17}$. En ce qui concerne la réaction des würzbourgiens aux objections, Bühler affirme simplement qu' « il n'était pas nécessaire de réagir aux objections contre l'introspection ${ }^{18} »$, tandis que Marbe et Messer s'arrêtent plus en détail sur un problème méthodologique de leurs recherches. Ils soulignent que même si ce qui est visé est le hic et nunc de la pensée, ce qui est rapporté par les sujets des expérimentations est le vécu passé de la pensée. Le hic et nunc de la pensée n'est néanmoins accessible que par une observation rétrospective qui, si possible, devrait avoir lieu immédiatement après l'expérience vécue de la pensée. Il existe une unanimité sur le fait qu'une introspection qui tenterait de se réaliser au moment même de la pensée entraverait le déroulement de celle-ci et pourrait même l'interrompre; en ce sens une observation de la pensée est précisément inopportune, confirment les chercheurs de Würzbourg. Brentano développait dans la Psychologie du point de vue empirique ce même argument en réagissant à Comte qui dénonçait une psychologie fondée sur l'idée d'un dédoublement du sujet en «l'organe observé et l'organe observateur $^{19} »$. La croyance qu'on pourrait penser et simultanément observer comment on

15. Pour une description plus détaillée du dispositif de recherche et de ses résultats, voir FrIEDRICH, 2008.

16. AsH, 1999, p. 69.

17. Depuis deux décennies on commence néanmoins à revaloriser l'introspection comme méthode d'investigation psychologique. Nous renvoyons au débat déclenché par Nagel dans son célèbre article «Quel effet cela fait, d'être une chauve-souris?» Dans ce texte, Nagel désigne comme déficit à combler en sciences cognitives la saisie objective de la phénoménalité des processus psychiques. À partir de là on trouve de plus en plus souvent des références à l'introspection au sein des sciences cognitives elles-mêmes. Ainsi, dans l'introduction du livre Naturaliser la phénoménologie, signée par Jean-Michel Roy, Jean Petitot, Bernard Pachoud et Francisco Varela, on peut lire : « [...] le sentiment est aujourd'hui de plus en plus répandu que la théorie cognitive a réagi de façon exagérée à l'introspectionnisme excessif qui a marqué les débuts de la psychologie, en cédant à son tour à un excès d'expérimentalisme » (Roy, Petitot, Pachoud et Varela, 2002, p. 19, voir aussi p. 9 et 16). Pourtant, et ce même ouvrage en témoigne, le scepticisme vis-à-vis de la méthode de l'introspection persiste.

18. BüHLER, 1907b, p. 305-306 (c'est nous qui traduisons les citations de cet auteur, sauf mention contraire).

19. Cомте, 1830, ici 1968, p. 29. 
pense, est déclarée par Comte chose simplement impossible: il s'en sert comme argument pour exclure la psychologie des sciences. Brentano rétorquait à cet argument:

«Mais, notez le bien, nous avons parlé de la perception interne et non de l'observation interne comme source première et indispensable de la psychologie. Ces deux notions sont loin d'être synonymes. Bien plus, c'est le caractère essentiel de la perception interne de ne jamais pouvoir se transformer en observation interne. [...] Conformément à une loi psychologique constante, nous ne pouvons appliquer notre attention à l'objet de la perception interne ${ }^{20}$.»

Comme l'objet de la perception interne ne peut pas être transformé en un objet de l'observation, c'est la mémoire qui servira en fin de compte de support, ce qui explique pourquoi Brentano parle d'une observation mnémonique ${ }^{21}$. En opérant cette même distinction entre perception interne et observation interne, Bühler rejette une autre objection à l'introspection qui, elle, postule qu'un emploi scientifique de cette dernière devrait se fonder sur une théorie de l'introspection. Pour Bühler, une telle demande est simplement circulaire et privée de sens. Les chercheurs de Würzbourg n'avancent pas sur la voie d'une psychologie dite phénoménologique qui tenterait de décrire comment se constitue cette expérience consciente de la pensée et comment elle pourrait être affinée. D'ailleurs le reproche leur a été fait de ne s'être jamais préoccupés d'analyser de manière fine et détaillée cet acte cognitif spécifique qu'est l'introspection, si utilisée par eux ${ }^{22}$. Pourtant un tel reproche signifierait mal comprendre l'objectif de leurs recherches. Leur but n'était pas de rendre compte d'une manière de plus en plus objective des données phénoménologiques, de la particularité de la conscience phénoménologique, comme cela semble être un des objectifs majeurs dans l'actuelle éclosion des études sur la conscience ${ }^{23}$.

En dépit de leur légitimité apparemment inébranlable, toutes les objections contre la méthode de l'introspection contrôlée, ultérieurement répétées au cours du Xx $\mathrm{X}^{\mathrm{e}}$ siècle, ratent d'une manière ou d'une autre leur cible, car ce qui est en question à Würzbourg ce n'est pas la méthodologie scientifique utilisée, ni la phénoménalité du psychique même (dans le langage moderne: les qualia de la conscience), mais la connaissance psychologique de la pensée. Bühler cherche à propos de la pensée quelque chose d'inconnu, de non encore déterminé qui ne sera accessible qu'à travers une analyse du vécu de la pensée, à l'intérieur d'une psychologie descriptive de la pensée. Sans ces données du vécu il n'existe, selon lui, que deux possibilités pour saisir ce qu'est la

20. Brentano, 1874, ici 2008, p. 42.

21. Voir Brentano, 1874, ici 2008, p. 53 et 61.

22. Ce reproche a été par exemple formulé par Pierre Vermersch qui tente d'aborder l'introspection comme « pratique concrète, c'est-à-dire comme acte psychologique effectivement mis en œuvre par un sujet singulier » (VERMERSCH, 1997, p. 11) et qui la transforme en objet central de l'analyse psychologique. Voir aussi Vermersch, 1999.

23. Ce but est aussi visé par Nagel quand il incite à développer une « phénoménologie objective » : «Outre son intérêt propre, une phénoménologie qui soit en ce sens objective permettrait aux questions portant sur le fondement physique de l'expérience de prendre une forme plus intelligible. Des aspects de l'expérience subjective qui recevraient ce genre de description objective pourraient être de meilleurs candidats pour des explications de type plus familier » (NAGEL, 1974, ici 1987, p. 404). Voir aussi Metzinger, éd., 1995. 
pensée. Soit le psychologue se fie à ses propres interprétations (non-contrôlées) de ce qu'il voit et pense de ces phénomènes, soit il utilise une des théories de la pensée déjà élaborées et proposées notamment à l'intérieur de la logique. En revanche, le but de Bühler est d'alimenter une théorie de la pensée à partir des recherches psychologiques sur ce phénomène, bref à partir d'un point de vue de psychologue.

\section{L'AMBIGUÏTÉ DU REPROCHE DE PSYCHOLOGISME}

C'est justement la relation entre logique et psychologie et la revendication par la psychologie d'une contribution propre à une théorie de la pensée qui semblent constituer l'arrière-fond, ce à quoi se réfèrent plus ou moins explicitement les würzbourgiens. On est donc tenté de situer leurs recherches dans un des débats les plus virulents en Allemagne en ce début du $\mathrm{Xx}^{\mathrm{e}}$ siècle, le débat autour du dit psychologisme. Il y a unanimité sur ce qui est visé dans ce débat, à savoir la surestimation de la psychologie en tant que science fondamentale pour les sciences et avant tout pour la logique et la philosophie. L'argument est bien connu: il serait impossible de déduire les lois de la pensée d'une analyse du fonctionnement empirique de la pensée. Car une telle analyse ne donne accès à la pensée que sous la forme des actes individuels de jugement ou de raisonnement, à la pensée comme processus doué d'un commencement et d'une fin dans le temps et dans l'espace, bref à la pensée en tant qu'événement individuel. La demande de Frege reformulée par Dummett dans le titre très parlant « L'expulsion des pensées hors de la conscience ${ }^{24} \gg$, signale un problème apparemment insoluble pour la psychologie empirique: comment saisir et expliquer par de telles analyses le contenu idéal et objectif de la pensée, existant et valide indépendamment du fait d'être pensé ou non par un individu? Ce qui est défendu dans ce débat par Frege, Husserl et d'autres, c'est la validité objective des lois logiques et l'affirmation de l'existence d'un type d'objets bien spécifiques, à savoir des objets idéaux.

Pour les psychologues, il semblait n'exister qu'une possibilité d'éviter ce reproche et on la trouve plus ou moins explicitement exploitée dans leurs recherches. Le reproche de psychologisme se trouve désamorcé par une répartition du travail, puisqu'il paraît parfaitement concevable de démarquer comme deux domaines de production de la connaissance bien différents, la psychologie de la logique. Dans une telle perspective, il revient à la psychologie d'étudier l'appareil psychique en tant que condition indispensable pour l'accomplissement de l'activité de pensée. Des approches psychogénétiques sont considérées comme propres à cette nouvelle science. Elles traitent la pensée comme produit aussi bien de l'évolution que d'apprentissages, en cherchant à donner des réponses à des questions comme celle-ci : comment l'espèce humaine avec son équipement biologique et psychologique a-t-elle acquis la capacité de penser la réalité ? Ce type de questions conduit à une analyse poussée des conditions d'ordre physico-psychologique, biologique et physiologique de la pensée, et renouvelle le paradigme d'une réalité double de l'homme, comme corps et comme âme. Une autre façon de réagir au reproche de psychologisme se trouve formulée dans la thèse complémentariste.

24. Dummett, 1991, p. 37. 
En acceptant les objections des philosophes et logiciens selon lesquelles le contenu de la pensée serait indépendant et non inférable des fonctions psychiques, nombre de psychologues, comme par exemple Stumpf et Twardowski, réagissent par une « redéfinition » de la tâche de la psychologie. Ainsi Stumpf insiste sur le fait qu'il faut clairement distinguer les fonctions psychiques qui constituent l'objet de la psychologie, des formations (Gebilde) dont font partie aussi bien les vérités logiques, les connaissances géométriques, les valeurs morales, les critères de beauté, etc. ${ }^{25}$ Une identification des deux rend le reproche de psychologisme justifié. Stumpf est d'accord sur le fait que la démonstration du caractère nécessaire et objectif des formations n'a pas besoin de la psychologie, puisque la preuve de l'évidence intuitive des propositions logiques, des valeurs éthiques, etc., revient exclusivement à la théorie de la connaissance. Pourtant, ce partage du travail ne signifie pas que ces formations sont à considérer comme des idées platoniciennes ayant un lieu d'existence ailleurs, ou comme des formes a priori de l'entendement ou de l'intuition, dont l'existence indépendamment des sensations et perceptions est affirmée par les successeurs de Kant (ledit criticisme) qui sont la cible d'élection de Stumpf ${ }^{26}$. Pour Stumpf, il n'existe aucun doute: ces formations (Gebilde) existent exclusivement comme contenus des fonctions psychiques ${ }^{27}$, comme d'ailleurs les formes a priori de l'intuition de Kant n'existent qu'à travers les perceptions du monde et sont immanentes à ces dernières. Cette affirmation en suscite nécessairement une autre, concernant l'objet de la psychologie qui, elle, aurait, selon Stumpf, à analyser la genèse (du contenu) de ces formations. Mais, attention, genèse veut dire ici une chose bien précise: ce terme signale selon Stumpf le fait que les fonctions psychiques sont une espèce de «support» de ce contenu. Le psychologue est donc celui qui montre par ses analyses que ce contenu comme aussi lesdites formes a priori de connaissance se trouvent dans nos jugements, ils sont immanents à eux et peuvent donc en être inférés (entnommen) ${ }^{28}$. Ainsi le contenu idéal (le contenu que constituent les formations) apparaît deux fois: une fois dans son évidence intuitive et à ce titre en tant qu'objet des philosophes, et une deuxième fois comme ce même contenu dans son immanence aux fonctions psychiques (à la pensée, au jugement), constituant sous cette forme-là l'objet du psychologue. La thèse complémentariste permet donc de redonner à la psychologie son rôle central en postulant qu'une logique, une esthétique, une éthique sans psychologie seraient une entreprise non sensée ${ }^{29}$.

Pourtant cette répartition du travail entre logique et psychologie fige une présupposition à laquelle on accorde encore aujourd'hui la plus grande importance. Il s'agit d'une certaine conception de l'objectivité des faits psychiques. Une objectivité qui se laisse déceler dans le fait qu'on cherchera à étudier le psychique soit comme condi-

25. STUMPF, 1906, ici 2006, p. 198: «La nécessité de distinguer les formations tant des phénomènes que des fonctions, de la manière la plus manifeste dans le domaine de la logique et celui de la théorie de la connaissance, doit s'imposer à la philosophie présente. » Voir aussi STUMPF, 1906, ici 2006, p. 174-175, 199, 201.

26. Voir notamment STUMPF, 1891.

27. STUMPF, 1906 , ici 2006, p. 199-200 et 209: « [...] que les formations ne sont pensables que comme contenus de fonctions psychiques. »

28. En ce qui concerne la manière d'analyser la genèse des concepts, voir STUMPF, 1891, p. 491.

29. Voir StumpF, 1906, ici 2006, p. 200. 
tion, soit comme support (lieu) des processus de pensée, de jugement, dont le contenu et son analyse feraient l'objet d'autres sciences. La thèse défendue ici est donc que le reproche de psychologisme auquel les psychologues se trouvent confrontés amène à une restriction de leur propre domaine d'investigation, en d'autres termes à une conception restreinte des phénomènes psychiques, à une délimitation nocive de ce qui est considéré comme un fait psychique et en conséquence déclaré objet de cette science. Bühler, de son côté, ne voit pas pourquoi le psychologue de la pensée ne pourrait pas chercher une confirmation expérimentale des thèses des philosophes. Tout au début de son habilitation, Bühler écrit que Husserl aurait instauré une méthode transcendantale déterminant le fonctionnement et le contenu des lois logiques et aurait posé en même temps la question de savoir ce qu'on pourrait en inférer à propos des phénomènes psychiques considérés comme leurs porteurs. Cette question oblige, selon Bühler, à réviser fondamentalement notre conception du psychique et à renoncer à l'idée d'une non-correspondance entre les lois logiques et les lois psychologiques de la pensée ${ }^{30}$.

Au premier abord, cette thèse de Bühler semble étrange car elle inverse la manière habituelle de réagir au reproche de psychologisme. Au lieu de délimiter le champ psychologique et de jouer la carte de la complémentarité, Bühler rebondit sur l'idée de la correspondance entre lois logiques et phénomènes psychiques de la pensée. Mais il est essentiel lorsqu'on lit Bühler de bien comprendre qu'il renonce en même temps à l'idée qu'on sait ce que sont les phénomènes psychiques de la pensée. Bühler déclare vouloir justement découvrir les « constituants psychologiques de la pensée » (psychologische Bestandstücke des Denkens) et non pas les présupposer. Cette volonté et son recours à la méthode de l'introspection contrôlée montre qu'il identifie la psychologie avec une science de la conscience. C'est la seule possibilité de faire de la psychologie de manière, selon lui, à préserver l'intention de contribuer en tant que psychologue à une théorie de la pensée ${ }^{31}$. C'est la raison pour laquelle il écarte une démarche qui se limite à une étude du psychisme comme condition ou support de la pensée dont le contenu est décrit par d'autres sciences. Cette manière de chercher une issue au débat entre psychologues et philosophes-logiciens n'a pas vraiment été prise au sérieux, parce que le reproche de psychologisme considère automatiquement comme vaines toutes les tentatives de participer à ce débat en tant que psychologue, autrement dit en ayant le projet de concevoir une théorie psychologique de la pensée qui ne soit pas seulement une théorie complémentaire à la théorie philosophique de la pensée ${ }^{32}$.

30. Voir BÜHLER, 1907b, p. 298-299.

31. Il faut rappeler que Husserl lui-même refuse de tels projets. Voir par exemple: HussERL, 1906-1907, ici 1998, p. 251-254: «c) La théorie de la connaissance n'est pas non plus une psychologie descriptive. »

32. Dans les études sur l'école de Würzbourg, cet objectif est de temps en temps évoqué. Ainsi MüNCH, 1997, déplore que Husserl n'a jamais répondu à la tentative de l'école de Würzbourg qui cherchait une confirmation expérimentale de ses thèses phénoménologiques. Voir aussi MiskiEwICZ, 2003, p. 229, qui mentionne que Bühler « a travaillé sur une confirmation expérimentale de ces postulats husserliens » et souligne aussi que ces tentatives n'ont pas retenu l'intérêt de Husserl. 
Cela montre une fois de plus que le terme de psychologisme prête à un malentendu ${ }^{33}$ et à une croyance prématurée qui suggère que le débat est clos par le fait que les psychologues ont accepté les critiques formulées à l'égard d'une psychologie de la pensée. En analysant dans ce qui suit les résultats de l'étude de Bühler, se pose donc la question suivante: qu'est-ce que Bühler découvre quant à la nature psychique de la pensée, quant à ces constituants psychologiques de la pensée, dans son habilitation publiée en 1907 et 1908 ?

\section{LES RÉSULTATS DES RECHERCHES ET LEUR INTERPRÉTATION}

\section{Une pensée sans représentations (Vorstellungen)}

Dans les manuels sur l'histoire de la psychologie, les résultats obtenus par les collaborateurs de Külpe sont souvent rapportés d'une manière abrégée, sous forme d'un slogan: «L'école de Würzbourg a démontré qu'il existe une pensée sans images, sans représentations. » Il est vrai, les procès-verbaux le montrent, que lorsque les sujets parlent de leurs vécus de la pensée, ils ne savent souvent plus si une représentation a été présente, tandis que ce qu'ils ont pensé, ils ont pu le déterminer avec assurance. La conclusion de Bühler est claire et ne laisse aucun doute: ce qui dans le processus de pensée apparaît à la conscience d'une manière aussi lacunaire, aussi sporadique, aussi hasardeuse que les représentations ne peut pas constituer la base de son contenu. Bühler conclut:

«Si le "quoi" de ma pensée peut être déterminé pour moi avant que je dispose des représentations mentales susceptibles par ailleurs de déterminer ce quoi, alors la pensée doit en tout état de cause être autre chose que ces représentations ${ }^{34}$.»

À Wundt, qui objectait que pour pouvoir penser à quelque chose, par exemple à une locomotive, je dois soit produire une représentation d'elle, soit utiliser un mot, Bühler rétorque que chaque chose peut être pensée en tant que quelque chose de déterminé sans la médiation d'une image ou d'un autre auxiliaire. On peut penser le concept d'un triangle ou d'un homme sans avoir besoin d'une seule image, d'une seule représentation.

« J'affirme au contraire qu'en principe tout objet peut être complètement et exactement pensé sans le secours de l'image. Je peux penser d'une manière entièrement déterminée et sans image cette nuance spéciale de bleu d'un tableau qui est pendu dans ma

33. Ce fait est de plus en plus souvent signalé, voir par exemple: FisETTE et FrÉChETTE, 2007 , p. 140. Nous ne partageons néanmoins pas tout à fait leur opinion, selon laquelle le reproche de psychologisme concernait exclusivement « la fondation de la logique sur la psychologie génétique ou physiologique » (FISETTE et FréchetTe, 2007, p. 144). L'école de Würzbourg est un bon exemple pour illustrer le fait que toutes les tentatives de prouver une correspondance (et non pas seulement une complémentarité) entre les lois logiques et la pensée comme phénomène psychique, même les tentatives entreprises à l'intérieur d'une psychologie descriptive, n'ont guère trouvé de reconnaissance.

34. BÜHLER, 1907 b, p. 327. 
chambre, pourvu seulement qu'il soit possible que cet objet me soit donné d'une autre manière que par les sensations ${ }^{35}$. »

Ce refus ferme d'une théorie représentationnelle de la pensée est articulé chez Bühler à une thèse qui le rapproche une fois de plus de la psychologie descriptive de Brentano et de ses élèves. Il s'agit de la thèse de l'immanence du psychique. Bühler a une conscience aiguë de la naïveté d'un projet référentiel de la conscience lorsqu'il écrit:

« Toutes les déterminations (caractéristiques) d'un objet que je connais, je ne les connais que dans et à travers les modifications de ma conscience, cela présente une affirmation qui est évidente en soi. Et la psychologie n'a qu'à faire avec ces modifications (de la conscience). Demander que le psychologue reste dans ces déterminations immanentes à la conscience, est la même chose que demander qu'il retourne aux choses ${ }^{36}$. »

Bühler convient qu'il est attirant de croire que dans notre pensée nous nous référons à quelque chose dont les déterminations seraient saisissables hors de la conscience. Pourtant, ce que les recherches ont montré, c'est que l'objet de la pensée est toujours contenu dans la pensée et qu'il serait trompeur de s'imaginer un objet qui transcende cette pensée, donc un objet dit réel qui serait «représenté » par la pensée. En refusant de prendre en compte dans l'explication d'un phénomène psychique la « transcendance de l'objet », Bühler défend une position proche de Husserl. On pensera à ce que Husserl écrit dans les Recherches logiques: « Du point de vue phénoménologique réel, l'objectivité elle-même n'est rien; car elle est, pour employer une expression générale, transcendante à l'acte ${ }^{37}$. " Ce que Mulligan rapporte au sujet de Husserl et de Wittgenstein est donc également valide pour Bühler, ce qui confirme une fois de plus la thèse de l'affinité des réflexions de Bühler avec celles de la philosophie autrichienne, thèse soutenue depuis des années par Mulligan.

«Husserl et Wittgenstein aiment souligner qu'une idée ou pensée (ou leur expression) est en rapport direct avec son objet et n'a pas besoin d'objet intermédiaire. Entre la pensée, et son objet, insistent Husserl (Cinquième recherche, appendice aux §11, §22) et Wittgenstein (Le Cahier bleu, p. 106) n'intervient aucune "ombre" de l'objet ${ }^{38}$. »

C'est dans ce contexte philosophique que les interprétations des recherches expérimentales de Bühler sur la pensée prennent tout leur sens. Mais en fin de compte, qu'est-ce que le quoi de la pensée si ce n'est pas une représentation? Qu'est-ce qui reste si le contenu de la pensée doit être circonscrit indépendamment de la représentation? Bref, qu'est-ce que la pensée? Trois choses sont caractéristiques de celle-ci, répond Bühler en 1907: la conscience de la règle, la conscience du rapport et les intentions. Nous ne pouvons ici détailler autant qu'il le faudrait ces trois « constituants des vécus de la pensée » (die Bestandstücke unserer Denkerlebnisse). Limitons-nous à une brève discussion de la conscience de la règle.

35. BÜHLER, 1907b, p. 321, cité par BuRLOUd, 1927, p. 123-124.

36. BÜHLER, 1907b, p. 355.

37. HuSSERL, 1901, ici 1962, § 11-20, p. 218.

38. Mulligan, 2001, p. 13. 


\section{La conscience de la règle}

À partir de ce que les sujets des expérimentations rapportent dans les procèsverbaux, Bühler caractérise la conscience de la règle comme la prise de conscience d'une méthode qui permet de résoudre une tâche, comme un savoir de la manière dont on aboutit en général à la résolution de ce type de tâches. C'est une pensée dans laquelle quelque chose que nous désignons du point de vue logique comme une règle vient à la conscience.

«C'est la prise de conscience d'une méthode pour accomplir une tâche. Dans les cas typiques, le phénomène ne contient pas seulement la notion de la manière dont cette tâche doit être accomplie, mais la notion d'une méthode générale valable pour toutes les tâches de même espèce; c'est une clef logique qui vient à la conscience ${ }^{39}$. »

Mais cette définition est insuffisante, car comme le dit Bühler, « je peux aussi simplement viser (meinen) une règle », et cette " pensée à une règle » (la pensée à propos d'une règle) n'est néanmoins pas à identifier avec la conscience de la règle. « L'objet de la conscience de la règle n'est pas la règle elle-même, mais le fait, l'objectivité qu'elle désigne, à laquelle on peut l'appliquer, dont on peut parfois l'inférer ${ }^{40}$. » C'est pour cette raison qu'il distingue soigneusement entre « la pensée à une règle » (Denken an eine Regel) et « la pensée de la règle ou dans la règle » (Denken einer Regel oder in einer Regel $)^{41}$. Tandis que la "pensée à une règle " fait référence à une règle sous forme d'un concept, d'une abstraction, d'un produit de la réflexion, la « pensée d'une règle » se réfère au monde des objets et des faits qui est pensé dans la règle. Cette conscience de la règle existe sous la forme d'une conscience de ce qui est général dans les différents cas; pourtant, pour connaître ce qui est général, le sujet ne l'a pas isolé ni abstrait dans les cas précédents. La règle est pensée en confrontation avec de nouveaux cas sans qu'une généralisation précédente ait eu lieu, sans que le sujet fasse référence à une règle. Dans ce sens Bühler parle d'une pensée de la règle même (sie selbst denken) qui n'est pas à confondre avec une pensée à la règle (une pensée à propos de la règle). Pour Bühler, un des cas les plus beaux de la conscience de la règle est la pensée d'une fonction mathématique:

«Les théorèmes familiers que nous utilisons dans une démonstration mathématique nous sont donnés le plus souvent sous la forme d'une conscience de la règle. Les cas les plus beaux et les plus clairs me semblent fournis par la pensée des fonctions mathématiques. On peut, par exemple, penser clairement et d'une façon sûre la

39. BÜHLER, 1907b, p. 335, cité par Burloud, 1927, p. 129. Comme exemple Bühler cite la réponse de Dürr: «Pouvons-nous par la pensée saisir l'essence de la pensée? Réponse de Dürr: Oui. (10”) Conscience qu'on soulève, là, une difficulté qui, en fait, n'existe pas. J'avais conscience de cette idée générale que les propositions où le même concept revient deux fois sous une forme déterminée ne présentent qu'une apparence de difficulté » (BüHLER, 1907b, p. 337, cité par BURLOUd, 1927, p. 129).

40. BüHLER, 1907 b, p. 339.

41. Pour marquer la différence entre les deux manières de "penser une règle », Bühler se base sur deux prépositions en allemand: an (à) et in (dans). J'ai décidé de garder ce marquage à l'aide des prépositions: tout en sachant que les expressions choisies par Bühler ne correspondent pas à la syntaxe française, elles permettent en effet de faire saisir la différence qu'il vise. 
décroissance en fonction du carré de la distance, sans déterminer les termes entre lesquels la distance existe et ce qui décroît; c'est réellement la fonction même qui est pensée et clairement présente à la conscience, tandis que ce à quoi elle se rapporte peut se réduire à quelque chose d'indéterminé, qui n'est pensé que comme un point de comparaison ou comme le substitut vide d'un contenu de relation ${ }^{42}$.»

Bühler va jusqu'à dire que la conscience de la règle est une pensée dans laquelle certains objets que le logicien désigne comme des lois sont pensés d'une manière adéquate. À titre d'autre exemple pour la conscience de la règle, Bühler cite « les qualités de la Gestalt », un phénomène fortement discuté durant cette période. Voici ce que Bühler développe à cet égard: en regardant les lignes d'une figure mathématique compliquée, je ne sais pas quoi faire au début avec ces lignes, et puis d'un coup je le sais, je le saisis. Qu'est-ce que je sais, qu'est-ce que j'ai saisi? - demande Bühler. "Apparemment le sens de la figure », et il poursuit: «[...] ce sens est en tout cas quelque chose d'idéel, dans beaucoup de cas rien d'autre que sa loi, même si cette loi n'est pas sa loi exacte, complète et précise, mais une règle brute de la constitution de la figure $^{43}$. » C'est là sans doute l'idée la plus importante pour Bühler. Cette loi de la figure nous apparait sous la forme d'une conscience de la règle. Grâce à cette conscience de la règle, l'image perceptive que j'ai de la figure est pénétrée par une lumière (eigenartige Durchleuchtung des sinnlichen Bildes). C'est la raison pour laquelle on dira à propos de ce qui se passe qu' « il me vient une lueur » (je commence à y voir clair). Bühler nous rappelle que quelque chose de semblable se passe, quand je « comprends » d'un coup la construction d'une machine ou le plan d'un bâtiment, mais le verbe comprendre est ici mis entre guillemets car cette compréhension se fait justement sur le plan perceptif, « je commence à y voir clair ». Cette métaphore visuelle souligne le fait que la conscience de la règle, étant constitutive pour la qualité de Gestalt, comme aussi pour la « compréhension » de la machine, n'est pas à confondre avec une connaissance de la règle qui pourrait être exprimée, explicitée, formulée par le sujet. Dans la majorité des cas, cette règle n'existe pas sous la forme d'une connaissance et pourtant elle est « consciente », d'où la capacité de l'individu de percevoir la Gestalt.

Bühler s'empresse de rapporter d'autres exemples. Les règles grammaticales peuvent également fonctionner comme une conscience de la règle. C'est le cas quand elles apparaissent (deviennent conscientes) au moment de l'expression langagière de nos pensées. Lorsque nous sommes en train de formuler une phrase, nous nous rendons compte que les règles grammaticales demandent que nous utilisions à cet endroit de la phrase une préposition, sans laquelle la phrase ne peut pas être terminée d'une manière correcte.

« Sur le trajet de la pensée à la phrase peuvent prendre place des états de conscience qui sont l'anticipation formelle de la phrase ou de son armature. Nous prenons conscience des règles grammaticales dans les cas où nous sommes incertains au sujet de leur application, et lorsque nous commettons ou quelque autre commet une faute contre elles ${ }^{44}$. »

42. BÜHLER, 1907b, p. 340-341, cité par BurLoud, 1927, p. 131.

43. BÜHLER, 1907 b, p. 341.

44. BüHLER, 1907b, p. 341, cité par Burloud, 1927, p. 131. 
Dans son étude sur Bühler, Toccafondi souligne que « le binôme Bühler-kantisme est entièrement défendable ${ }^{45} »$. Ce modèle actif de l'esprit qu'on trouvera exprimé dans les réflexions de Bühler sur la conscience de la règle est clairement inspiré de Kant. Bühler lui-même soutient que l'affirmation gnoséologique de Kant, selon laquelle l'entendement est la capacité des règles, peut être prouvée sur le plan psychologique. C'est le paragraphe sur le schématisme de la raison qui retient notamment l'intérêt de Bühler, puisque ce que Kant démontre quant à la manière dont le contenu d'un concept peut être pensé correspond à ce qu'il a découvert dans ses expérimentations.

« Ainsi, quand je dispose cinq points les uns à la suite des autres ....., c'est là une image du nombre cinq. Au contraire, quand je ne fais que penser à un nombre en général, qui peut être cinq ou cent, cette pensée est la représentation d'une méthode pour représenter une multitude (par exemple, mille) dans une image, conformément à un certain concept, plutôt que cette image même, qu'il me serait difficile, dans le dernier cas, de parcourir des yeux et de comparer au concept. Or, c'est cette représentation d'un procédé général de l'imagination pour procurer à un concept son image que $\mathrm{j}$ 'appelle le schème de ce concept ${ }^{46}$.»

Cette idée des schèmes (des consciences des règles) qui sont dans la perception ou dans la parole, et sans lesquels les qualités de la Gestalt comme phénomènes perceptifs ou encore notre capacité d'engendrer un nombre infini de phrases ne pourraient pas être attestées ni expliquées, reste une idée centrale pour Bühler dans ses travaux ultérieurs, même si cette idée ne se trouve apparemment plus au centre de ses préoccupations.

\section{La conscience d'un ordre psychique des objets et la conscience des sphères}

Il est plutôt difficile d'attribuer aux recherches de Bühler sur la psychologie de la pensée une place bien déterminée dans son œuvre. D'une part il n'est jamais revenu sur ces recherches, il ne les cite dans ses textes ultérieurs que très peu, d'autre part son intérêt pour cette problématique ne s'est jamais totalement éteint. Ainsi il reprend après le décès d'Oswald Külpe en 1916 la direction de la thèse de sa future femme, Charlotte Malachowski ${ }^{47}$, qui a été écrite dans le prolongement direct des travaux de Würzbourg, comme d'ailleurs aussi la thèse de Karl Popper ${ }^{48}$, soutenue également sous la direction de Bühler en 1928 à Vienne. Ce sont les faits qui se laissent constater sur la « surface » de son œuvre, cependant le lecteur attentif retrouvera également son "idée centrale » de 1907 et 1908 dans les travaux considérés comme ses œuvres maîtresses, à savoir La Crise de la psychologie (1927) et La Théorie du langage (1934). Dans ces travaux, Bühler évoque l'existence d'une « conscience de l'ordre » et d'une « conscience des sphères » dont la description sera rapportée ci-dessous avant que, dans la dernière partie de ce texte, ne soient discutées les conséquences de la découverte de ces constituants psychologiques de la pensée et de la parole.

45. TOCCAFONDI, 1999, p. 293.

46. KANT, 1787, ici 1993, p. 152.

47. Voir Charlotte BüHLER, 1918.

48. Voir Popper, 1928. 
Déjà dans ses recherches sur la pensée, il évoque un « ordre psychique des objets ${ }^{49}$ ». Il s'agit comme avec la conscience de la règle d'une donnée phénoménologique, d'un fait présent ou attestable dans le vécu de la pensée. Bühler le dit ainsi : c'est à travers la place que les objets ont dans un ordre conscient du monde que se fait la pensée d'un objet concret du monde. Dans cet ordre psychique du monde, il y a une place marquée et vide pour n'importe quel objet et penser le monde consiste dans la majorité des cas à se référer à cette place vide. Bühler utilise dans ce contexte le terme de "schème de représentation » (Darstellungsschema) ${ }^{50}$ dans lequel il y a une place marquée à laquelle ce qui est pensé est rapporté. Ce qui est conscient pour le sujet est quelque chose comme un ordre dans lequel le « quoi » de la pensée, tout ce à quoi nous pouvons penser comme à quelque chose de déterminé, a une place et est $s u$ à travers cet ordre. Il termine ces réflexions par la considération suivante: "Cette manière d'englober, de saisir par la pensée ce qui est présent sur le plan perceptif grâce à la détermination de sa place dans un ordre est un cas spécifique de la détermination structurelle dans le domaine phénoménal ${ }^{51}$. » Et cet ordre, ce schème de représentation que nous connaissons encore peu devrait, selon lui, constituer un des objets centraux de la psychologie.

Un autre exemple pour un tel « outil » de mise en ordre, comme on pourrait aussi désigner les schèmes de représentation, se trouve dans la Théorie du langage. La question qui est posée dans la deuxième partie rappelle clairement les préoccupations de sa jeunesse: «Qu'est-ce que le locuteur et l'auditeur ont présent comme visée ou comme représentation lorsqu'ils utilisent des signes linguistiques comme "cheval”, etc.? » Qu'est-ce qui est ressenti dans l'emploi pratique des mots? Un stade intermédiaire, répond Bühler. Un stade intermédiaire est ce que les psychologues de la pensée ont identifié lorsqu'ils ont voulu mettre en évidence l'expérience que le locuteur a lorsqu'il utilise des signes linguistiques. Qu'est-ce que ce stade intermédiaire? Ce stade intermédiaire est ce stade bien connu par tous les locuteurs de n'importe quelle langue, dans lequel un mot que nous prononçons n'éveille plus en nous l'expérience vivante de son étymon. Dans ce stade l'étymon n'est plus vivant, il est « estompé » ou mort, et Bühler poursuit:

« Sur les quatre-vingt dix millions de locuteurs qui pratiquent aujourd'hui l'allemand, il n'y en a peut-être pas un sur dix-mille qui sache ce qu'ont jadis désigné étymologiquement des termes comme Pferd, "cheval", Kuh, "vache", Schaf, "mouton", Gans, "oie". Et pour celui qui le sait, cela reste un savoir froid, qu'il ne ressent aucunement dans quelque emploi pratique des mots que ce soit $^{52}$. »

Mais qu'est-ce qui est présent au locuteur quand il prononce le mot « cheval », si ce n'est pas l'étymon qui permettra de saisir ce qui dans la réalité est représenté par le

49. Voir BüHLER, 1907b, p. 357-358.

50. Soulignons qu'il s'agit bien du terme de Darstellung et non pas de Vorstellung que Bühler utilise ici. Le lecteur averti sait que la langue allemande fait une distinction entre Vorstellung (représentation en tant qu'image mentale) et Darstellung (représentation du monde par des signes de tout genre), une distinction que le français ne permet pas de reproduire. D'ailleurs, le terme de Darstellung devient chez Bühler central pour sa théorie du langage.

51. BÜHLER, 1927, ici 1978, p. 117.

52. BÜHLER, 1934, ici 2009, p. 344. 
mot? Bühler se réfère ici à sa femme, Charlotte, qui a utilisé dans sa thèse de 1918 le terme de « sphères ${ }^{53}$ » pour conceptualiser les résultats de ses recherches sur la psychologie de la pensée. Ces dernières avaient clairement montré que la « chose » visée dans un cas particulier par le locuteur qui utilise le mot « cheval » est séparée des autres par sa « sphère ».

« Le "cheval", pour en rester à cet exemple, relève en gros dans mon stock de connaissances de la sphère "animaux", ou "animaux domestiques", ou "animaux utilitaires". Et de telles mises en ordre par sphères se font encore sentir dans le vécu quand toute réalisation concrète d'image de chose est absente ${ }^{54}$. »

Cette dernière remarque rappelle ce que Bühler a évoqué par rapport à la conscience de la règle, elle est pensée sans qu'une représentation du monde soit produite, sans qu'une inférence ou une abstraction soient réalisées par les individus, et pourtant un savoir du monde est produit, un savoir qui a une particularité bien spécifique.

\section{Le concept bühlerien de savoir actuel (ou présent)}

Dans ses recherches à Würzbourg, Bühler attire déjà l'attention sur ce fait qu'il désigne d'abord comme «le savoir immédiatement». De quoi s'agit-il? D'un fait, attesté dans ses recherches empiriques sur la pensée, consistant dans la capacité du sujet de savoir immédiatement ce qu'il a pensé, ce qui a été inclus dans sa pensée, sans devoir rechercher un contenu auquel la pensée se réfère. On aura remarqué la récurrence de cette idée dans ce qui vient d'être discuté: les schèmes de représentation me font savoir la chose sans que je sois obligé de me la représenter, de même que l'utilisation quotidienne des mots ne présuppose pas que je connaisse son étymon, ce à quoi il se réfère. Un des énoncés cités par Bühler à partir des procès-verbaux de Würzbourg donne une très bonne illustration de ce type de savoir. Il peut être renvoyé dos-à-dos avec un autre énoncé provenant des Recherches philosophiques de Wittgenstein:

«Külpe F12: J'ai pensé au scepticisme antique (mot scepticisme intérieurement prononcé); beaucoup de choses étaient contenues là-dedans; j'ai eu un instant présent à l'esprit tout le développement du scepticisme en trois périodes ${ }^{55}$. »

« Je veux me souvenir d'une mélodie et elle ne me revient pas à l'esprit; soudain, je dis: "Maintenant je sais", et je la chante. Que s'est-il passé pour que soudain je la sache? Elle ne peut certainement pas m'être revenue à l'esprit dans son intégralité à ce moment-là ! - Tu diras peut-être: "C'est un certain sentiment, le sentiment qu'elle est maintenant là." - Mais y est-elle vraiment? [...] On peut naturellement dire: Si quelqu'un dit avec conviction que maintenant il sait la mélodie, à cet instant-là, elle est (d'une certaine façon) intégralement présente à son esprit - et c'est là une explication des mots : "La mélodie est intégralement présente à son esprit ${ }^{56}$ ". »

53. Ce terme de « sphère » a été déjà utilisé à Würzbourg, on le trouve développé chez Ach et Marbe. 54. BÜHLER, 1934, ici 2009, p. 349.

55. BÜHLER, 1907b, p. 346, cité par BurLOUd, 1927, p. 134.

56. WitTGENSTEIN, 1953, ici 2004, § 184, p. 117-118. 
L'expression «présent à l'esprit» pourrait susciter un commentaire mentaliste. Cependant, en la confrontant avec l'énoncé de Wittgenstein, l'intention est bien d'éviter ce soupçon, qui pour les lecteurs des Recherches philosophiques doit être immédiatement rejeté. Appliquons donc à l'énoncé de Külpe le même étonnement, cette même impression d'être mis devant un paradoxe que celle que Wittgenstein veut éveiller chez le lecteur, et que Bühler tente de prendre au sérieux par une interprétation nonmentaliste de ses recherches. Il est vrai qu'à la lecture de ces deux énoncés, on est tout de suite tenté de s'exclamer: comment est-il possible que tout le développement du scepticisme philosophique en trois périodes, ou la mélodie dans sa totalité, soient présents à l'esprit? Impossible, inimaginable! Mais qu'est-ce qui est alors visé par l'expression «présent à l'esprit»? Bühler répondrait probablement qu'il s'agit de la capacité du sujet de produire un savoir immédiat, qui se base sur des « consciences » bien spécifiques (la conscience de la règle, la conscience d'un ordre des choses, la conscience des sphères des mots).

Mais il est essentiel, lorsqu'on lit cette réponse, de ne pas oublier que pour Bühler ces « consciences » ne sont pas à confondre avec un produit de la réflexion. Ce n'est pas une psychologie de la réflexion qui est visée et pratiquée par lui. La conscience de la règle, la conscience de l'ordre psychique du monde, les sphères des mots ne sont pas à confondre avec l'expérience consciente qui a été utilisée pour les découvrir, elles n'existent pas sous une forme consciente, ou comme le dit Bühler : « [...] la conscience de la règle n'est pas un produit artificiel de l'analyse ultérieure des vécus ${ }^{57}$. » La pensée « paradoxale » de Wittgenstein nous semble de nouveau utile pour pouvoir prendre la mesure du refus d'une psychologie de la réflexion tel qu'il est mis en œuvre par Bühler:

« Lorsque je repense ensuite à cette expérience, j'ai le sentiment que ce qu'il y a en elle d'essentiel est "l'expérience d'une influence", d'une connexion entre les phénomènes par opposition à une simple simultanéité entre eux. Mais, en même temps, il n'y a aucun phénomène vécu que je nommerais "expérience de l'influence". Je dirais volontiers que j'ai eu l'expérience du "parce que", pourtant, il n'y a aucun phénomène que je nommerais "expérience du parce que" ${ }^{58}$. "

Bühler serait d'accord avec cette formulation de Wittgenstein. D'ailleurs, nous avons montré ci-dessus une interprétation semblable: en percevant la qualité de la Gestalt, j'ai l'expérience vécue d'une règle, pourtant, en même temps, il n'y a aucun phénomène qu'on pourrait appeler l'expérience vécue de la règle. Déjà en 1908, dans la deuxième partie de son habilitation, Bühler décrit cette expérience vécue qui n'en est pas vraiment une en exposant son idée de l'existence d'un schème syntaxique vide:

«Lorsque nous pénétrons une construction phrastique compliquée, il s'agit d'un savoir à propos de sa structure grammaticale, nous savons quelque chose des relations qui existent entre les parties séparées de la forme totale. Cela se produit aussi lorsque nous parlons nous-mêmes, par exemple lorsque nous débutons une incise par quand et qu'à

57. Voir BÜHLER, 1907b, p. 339.

58. Wittgenstein, 1953, ici 2004, §176, p. 114. 
la fin de la subordonnée, nous nous interrompons brusquement ; nous prenons alors conscience que nous avons attendu quelque chose; il ne s'agit pas seulement d'un complément matériel, mais aussi d'un complément grammatical, nous attendons une proposition principale. Dans tous ces cas, nous prenons conscience sous forme séparée de ce qui, incidemment et sans qu'on y prête spécialement attention, sert toujours ou presque toujours d'intermédiaire entre les pensées et les mots, en l'occurrence un savoir quant à la forme phrastique et quant à la relation des constituants phrastiques entre eux, quelque chose qu'il faut considérer comme une expression directe des règles grammaticales qui sont vivantes en nous ${ }^{59}$. »

Il est donc bien certain que selon Bühler la conscience de la règle, l'ordre psychique du monde et les sphères des mots sont vivants en nous et leur seule forme d'existence (expression directe) sont la perception de la Gestalt, la connaissance de l'objet et l'emploi du mot. C'est pour cette raison que Bühler les désigne comme des constituants vécus de la pensée et de la parole et affirme que, sur le plan de leur fonctionnement, on devrait les identifier au savoir, mais il ajoute aussitôt que ce savoir ne devrait pas être compris comme une disposition.

Cette introduction du terme de savoir est extrêmement intéressante car on sait que ce même terme joue un rôle central dans la critique du mentalisme développée par Wittgenstein. Le refus du dispositionnalisme rapproche également ces deux auteurs. Bühler remarque dans son habilitation qu'au sens courant, le savoir est souvent identifié avec ce qui se trouve stocké dans la mémoire, on lui accorde une présence virtuelle dans l'esprit qui permettrait de prédéterminer (causer) le fonctionnement chaque fois concret de la pensée. Pour éviter cette confusion, Bühler propose de distinguer de ce savoir potentiel le savoir actuel. Cependant ce terme peut également prêter à confusion, si on comprend par le savoir actuel les occurrences ou performances chaque fois nouvelles et ponctuelles d'un savoir qui existerait exclusivement sous ces formes-là. Le savoir actuel dont parle Bühler ne serait cependant pas à comprendre à l'intérieur du débat qui l'identifie soit avec une disposition soit avec une performance ${ }^{60}$. Voici ce que Bühler tente de clarifier à travers un exemple: quand j'entends un mot, j'ai une impression sensorielle de ce mot et en même temps il m'est donné un sens, non pas parce que je me représente quelque chose, mais parce qu'un savoir m'est présent quand j'entends le mot ${ }^{61}$.

Nous sommes enclins à affirmer que ce savoir dont parle Bühler est « de l'ordre de l'être plutôt que de l'avoir », et cette différence semble extrêmement importante et se reflète dans l'adjectif présent que Bühler attribue également à ce savoir. L'individu n'a pas à l'esprit ce savoir, « il est présent» veut dire que ce savoir a une présence dans sa perception du monde, dans sa pensée du monde, dans son parler du monde. Bühler

59. BüHLER, 1908a, p. 86 (traduction Didier Samain; c'est nous qui soulignons).

60. Voir sur ce débat CHAUviRé, 2003, et son article dans le présent ouvrage (supra, p. 21-34). Cette alternative se trouve également formulée par Wittgenstern, 1953, ici 2004, p. 99: «Quand sais-tu jouer aux échecs? Toujours? Ou au moment où tu joues un coup? Et chaque fois que tu joues un coup, sais-tu tout du jeu? - Comme il est étrange que savoir jouer aux échecs requière si peu de temps et qu'une partie d'échecs en prenne tellement. »

61. Voir BüHLER, 1907b, p. 363. 
caractérise aussi ce savoir comme le pouvoir de savoir à propos de quelque chose (ich kann wissen um etwas). On pourrait recourir à une comparaison avec la notion de médium dans la tradition de la pensée magique; une comparaison qui semble néanmoins pertinente pour saisir ce qu'est ce savoir actuel et présent. Le médium fait qu'un monde - dans le cas d'une séance spiritiste, le monde de l'au-delà - devient directement accessible. Transposé au savoir actuel, on pourrait dire qu'à travers ce savoir un monde devient aussi directement accessible, et cela justement parce qu'il est su.

Nous l'avons vu, Bühler a mis au centre de ses recherches à Würzbourg ces constituants de la pensée dont la caractéristique essentielle pourrait être maintenant résumée comme suit: ils existent exclusivement sous forme de vécu, en constituant en même temps un être (une présence) conceptualisé par Bühler à l'aide du terme de savoir actuel. Bühler insiste sur le fait qu'admettre que ces constituants vécus de la pensée forment la présence (l'être) du savoir, c'est affirmer en même temps qu'ils opèrent exclusivement d'une façon immanente ou interne ${ }^{62}$. Il est vrai qu'il y a un statut de l'être, de la présence qui est accordé au savoir actuel, mais en même temps Bühler ne cesse de s'opposer à l'idée que ce savoir présent a un lieu, a un siège, qu'il soit « en haut» du ciel platonicien ou « en bas» dans les structures cérébrales. Ce sont des thèses qu'il a rigoureusement contestées. On ne trouvera donc pas les constituants de la pensée « dans l'esprit », comme il est présupposé pour les dispositions, le langage de la pensée ou les structures neurophysiologiques. Ils ne se prêtent pas à la naturalisation car ils « sont, comme le disait Bühler, vivants en nous » et leur pouvoir trouve son expression (sa seule existence) dans les caractéristiques bien spécifiques de la perception, de la pensée et de la parole que nous avons esquissées ci-dessus. Le but de Bühler a été de « sauver» la pensée comme objet de la psychologie en produisant une connaissance de celle-ci qui démontre son fonctionnement en tant que phénomène psychique. Il s'oppose alors à une analyse du fonctionnement de la pensée en tant que condition ou support (fonction) d'un contenu qui existe hors du psychique. Il semble que Bühler était conscient de la difficulté que posait ce projet: rester psychologue en étant capable d'éviter le mentalisme localisationniste, qu'il soit empirique ou transcendantal. C'est peut-être pour cette raison que Bühler s'est intéressé dès le début de sa carrière au langage.

$\mathrm{Au}$ cours $\mathrm{du} \mathrm{xx}^{\mathrm{e}}$ siècle, les analyses du langage ont succombé moins facilement au soupçon mentaliste, ce qui a souvent eu pour conséquence, néanmoins, une incommensurabilité entre théories linguistiques et théories psychologiques du langage. C'est notamment dans sa Théorie du langage (1934) que Bühler dégage dans le fonctionnement du langage un pouvoir (eine Leistung) tout à fait semblable à celui du savoir présent ${ }^{63}$. De ce point de vue, il y a une continuité dans l'œuvre de Bühler: celui-ci ne se transforme pas en linguiste pour traiter le langage, mais il existe chez lui une cohérence tout à fait convaincante entre théorie de la pensée et théorie du langage, une cohérence fondée sur un anti-mentalisme qu'il n'a cessé de rechercher tout au long de sa carrière.

62. Nous reformulons ici une affirmation de CHAuviRÉ, 2003, p. 293, quant à la conception des would-be de Peirce: « [...] c'est d'une façon immanente ou interne que les would-be de Peirce opèrent dans le monde de l'existence, et non du haut d'un ciel platonicien. »

63. Voir Friedrich, 2009. 


\section{LISTE DES RÉFÉRENCES}

Ash (Mitchell G. ), 1999, « Die Würzburger Schule. Kontext, Praxis, Rezeption », dans JAHNKE (Wilhelm) et SchNeIDer (Wolfgang), éd., Hundert Jahre Institut für Psychologie und Würzburger Schule der Denkpsychologie, Göttingen/Bern/Toronto/Seattle, Hogrefe, p. 52-72.

Brentano (Franz), 1874, Psychologie vom empirischen Standpunkt; Psychologie, du point de vue empirique, trad. franç. Maurice de Gandillac, Paris, Vrin, 2008.

BÜHLER (Charlotte), 1918, Über Gedankenentstehung. Experimentelle Untersuchungen zur Denkpsychologie, Leipzig, Barth.

BÜHLER (Karl), 1907a, « Remarques sur les problèmes de la psychologie de la pensée », Archives de psychologie, t. VI, p. 376-386.

BüHLER (K.), 1907b, « Tatsachen und Probleme zu einer Psychologie der Denkvorgänge: I. Über Gedanken », Archiv für die gesamte Psychologie, t. X, p. 297-365.

BÜHLER (K.), 1908a, «Tatsachen und Probleme zu einer Psychologie der Denkvorgänge: III. Über Gedankenerinnerungen», Archiv für die gesamte Psychologie, t. XII, p. 24-92.

BüHLER (K.), 1908b, « Tatsachen und Probleme zu einer Psychologie der Denkvorgänge : Antwort auf die von Wundt erhobenen Einwände gegen die Methode der Selbstbeobachtung an experimentell erzeugten Erlebnissen », Archiv für die gesamte Psychologie, t. XII, p. 93-122.

BüHLER (K.), 1927, Die Krise der Psychologie, rééd. Francfort-sur-le-Main/Berlin/Vienne, Ullstein, 1978.

BüHLER (K.), 1934, Sprachtheorie. Die Darstellungsfunktion der Sprache; Théorie du langage. La fonction représentationnelle du langage, éd. et trad. Didier SAMAIN et Janette FrIEDRICH, Marseille, Agone, 2009.

Burloud (Albert), 1927, La Pensée d'après les recherches expérimentales de H.-J. Watt, de Messer et de Bühler, Paris, Alcan.

Chauviré (Christiane), 2003, «Quand savoir, c'est (savoir) faire », dans ID., Le Grand miroir : essais sur Peirce et sur Wittgenstein, Besançon, Presses universitaires franc-comtoises, p. 278-293.

Cомтe (Auguste), 1830, Cours de philosophie positive, rééd. dans Euvres d'Auguste Comte, Paris, Anthropos, t. I, 1968.

Dilthey (Wilhelm), 1910, «Abgrenzung der Geisteswissenschaften»: «Délimitation des sciences de l'esprit », dans ID., L'Édification du monde historique dans les sciences de l'esprit (Euvres, t. III), trad. franç. Sylvie Mesure, Paris, CERF, 1988, p. 31-39.

Dummetт (Michael), 1991, Les Origines de la philosophie analytique, Paris, Gallimard.

Eschвасн (Achim), 1997, «Karl Bühler und die Würzburger Schule », Brentano-Studien, n 7 , p. 237-254.

FisetTe (Denis) et Fréchette (Guillaume), 2007, « Le legs de Brentano », dans ID., dir., À l'école de Brentano. De Würzbourg à Vienne. Husserl, Stumpf, Ehrenfels, Meinong, Twardowski, Marty, Paris, Vrin, p. 13-160.

FrIEDRICH (Janette), 2008, « La psychologie de la pensée de l'école de Würzbourg. Analyse d'un cas de marginalisation ", dans KaIL (Michel), dir., Marges et marginalisations dans l'histoire de la psychologie, $\mathrm{n}^{\circ}$ spéc. de L'Homme et la société, $\mathrm{n}^{\circ}$ 167-169, p. 251-278.

Friedrich (J.), 2009, « Présentation », dans BüHLER, 1934, ici 2009, p. 21-58.

HusSERL (Edmund), 1901, Logische Untersuchungen; Recherches logiques, trad. franç. Hubert ELI et al., t. II, part. II, Paris, Presses universitaires de France, 1962.

HuSSERL (E.), 1906-1907, Einleitung in die Logik und Erkenntnistheorie. Vorlesungen 1906-1907; Introduction à la logique et à la théorie de la connaissance, Cours (1906-1907), trad. franç. Laurent Joumier, Paris, Vrin, 1998. 
JAHNKE (Wilhelm) et SCHNEIDER (Wolfgang), 1999, Hundert Jahre Institut für Psychologie und Würzburger Schule der Denkpsychologie, Göttingen/Bern/Toronto/Seattle, Hogrefe.

Kant (Emmanuel), 1787, Kritik der reinen Vernunft; Critique de la raison pure, $2^{\mathrm{e}}$ éd., trad. franç. A. Tremesaygues et B. Pacaud, Paris, Presses universitaires de France, 1993.

Metzinger (Thomas), éd., 1995, Conscious experience, Thorverton, Imprint Academic.

Miskiewicz (Wioletta), 2003, «Husserl contre psychologisme et cognitivisme», dans FisetTe (Denis) et LaPoINTE (Sandra), dir., Aux origines de la phénoménologie. Husserl et le contexte des « Recherches logiques », Paris, Vrin, p. 221-240.

Muldigan, (Kevin), 2001, «De la philosophie autrichienne et de sa place», dans Mulligan (Kevin) et Cометті (Jean-Pierre), dir., La Philosophie autrichienne de Bolzano à Musil. Histoire et actualité, Paris, Vrin, p. 7-25.

MüNCH (Dieter), 1997, « Edmund Husserl und die Würzburger Schule », Brentano Studien, n 7 , p. 89-122.

NAGEL (Thomas), 1974, «Quel effet cela fait, d'être une chauve-souris? », rééd. dans HofSTADTER (Douglas) et Dennett (Daniel), dir., Vues de l'esprit. Fantaisies et réflexions sur l'être et l'âme, Paris, InterEditions, 1987, p. 391-404.

POPPER (Karl), 1928, Zur Methodenfrage der Denkpsychologie, Dissertation eingereicht zur Erlangung des Doktorgrades der philosophischen Fakultät der Universität Wien, Sommersemester.

Roy (Jean-Michel), Petitot (Jean), Pachoud (Bernard) et Varela (Francisco), 2002, « Combler le déficit: introduction à la naturalisation de la phénoménologie », dans Naturaliser la phénoménologie. Essais sur la phénoménologie contemporaine et les sciences cognitives, Paris, CNRS Éditions, p. 3-100.

Ryle (Gilbert), 1949, La Notion d'esprit. Pour une critique des concepts mentaux, rééd. Paris, Payot, 2005.

StumpF (Carl), 1891, «Psychologie und Erkenntnistheorie», dans Abhandlungen der philosophisch-philologischen Classe der Königl. Bayerischen Akademie der Wissenschaften, München, Verlag der Akademie, vol. XIX, part. II, p. 465-516.

STUMPF (C.), 1906, «Zur Einteilung der Wissenschaften » : « De la classification des sciences », dans ID., Renaissance de la philosophie. Quatre articles, éd. et trad. Denis FisetTe, Paris, Vrin, 2006, p. 169-254.

TocCAFondi (Fiorenza), 1999, « De Karl Bühler à Karl R. Popper », Philosophiques, t. XXVI, $\mathrm{n}^{\circ} 2$, p. 279-300.

Vermersch (Pierre), 1997, «L'introspection comme pratique », Expliciter, n 22, p. 1-19.

Vermersch (P.), 1999, «Introspection as Practice », Journal of Consciousness Studies, vol. VI, $\mathrm{n}^{\circ} 2-3, \mathrm{p} .17-42$.

VoNK (Frank), 1992, Gestaltprinzip und abstraktive Relevanz: eine wissenschaftshistorische Untersuchung zur Sprachaxiomatik Karl Bühlers, Münster, Nodus.

Wittgenstein (Ludwig), 1953, Philosophische Untersuchungen; Recherches philosophiques, trad. franç. Françoise DASTUR et al., Paris, Gallimard, 2004.

Wundt (Wilhelm), 1896, Grundriss der Psychologie, rééd. Leipzig, Körner, 1918.

Wundt (W.), 1907, « Über Ausfrageexperimente und über die Methoden zur Psychologie des Denkens », Psychologische Studien, ${ }^{\circ}$ 3, p. 301-360.

WundT (W.), 1908, « Kritische Nachlese zur Ausfragemethode », Archiv für die gesamte Psychologie, t. XI, p. 445-459. 